\title{
24-Hour Sleep/Wake Patterns in Healthy Elderly Persons
}

\author{
Barbara D. Evans and Ann E. Rogers
}

\begin{abstract}
The purpose of this study was to examine the 24-hour sleep/wake patterns of healthy elderly persons. Data was obtained from 14 elderly subjects who wore a wrist actigraph for 48 hours and completed an activity diary during the monitoring period. Although subjects spent slightly more than 7.5 hours in bed at night, they were asleep for just over 6 hours. Subjects did not have trouble falling asleep, but once asleep, had trouble remaining asleep. All subjects took one or more naps during the recording period, but daytime naps composed only a small fraction of their total sleep time. Total duration of daytime sleep averaged 59.8 minutes.

Copyright (C) 1994 by W.B. Saunders Company
\end{abstract}

$\mathbf{S}^{\mathrm{L}}$ LEEP IS A universal and vital human function. Assisting patients to receive adequate rest is a core component of nursing. Nursing strategies used to promote sleep have frequently been based on trial and error instead of on empirical evidence. For example, daytime naps are often discouraged in order to improve nighttime sleep. However, the appropriateness of this practice with elderly persons or younger individuals has not been tested. Furthermore, the question of whether daytime naps are the cause or the consequence of disturbed nighttime sleep remains unanswered.

\section{BACKGROUND}

One of the most consistent changes observed in the sleep of the aged is a progressive reduction and, in some cases, the total disappearance of the deepest levels of sleep (stages 3 and 4). The percentage of Stage 1 sleep (the lightest stage of sleep) almost doubles during old age, increasing from $8 \%$ to $15 \%$ of nocturnal sleep time (Bliwise, $1989 \mathrm{~b}$ ). Sleep efficiency or the amount of time asleep divided by the amount of time in bed, decreases with aging. Elderly persons awaken more often at night and stay awake longer than younger persons, sleeping only $70 \%$ to $80 \%$ of the time that they are in bed (Prinz, 1977). Therefore, the elderly often spend more time in bed in order to get the same amount of sleep as a younger person (Pressman \& Fry, 1988). The incidence of sleep apnea increases with age and is a frequent cause of sleep disruption in the elderly (Bliwise, 1989b; Pressman \& Fry, 1988). Serious problems can arise when the apneic episodes are frequent enough and/or long enough to cause hypoxemia, hypercapnia, and cardiac arrhythmias. Other potential consequences of sleep apnea include systemic and pulmonary hypertension, stroke, excessive daytime sleepiness, and altered cognitive function (Bliwise, 1989b). Periodic leg movements (PLMs) are also more frequent in elderly persons (Bliwise, 1989b). Although PLMs often occur in association with sleep apnea, PLMs can occur in persons who do not have any other sleep disorder. An individual with PLMs may be asymptomatic or may have significant complaints of disrupted and restless sleep (Bliwise, 1989b).

Although daytime napping is considered by some to be a natural adaptive response to the lighter and more fragmented nighttime sleep of the elderly, other researchers believe that napping contributes to this impaired sleep (Bliwise, Pursley, \& Dement, 1988). Daytime napping is common among both institutionalized and noninstutionalized elderly person (Ancoli-Israel, Kripke, Jones, Parker, \& Hanger, 1991; Hayter, 1983; Metz \&

From the Rochester General Hospital, Rochester, NY, and the School of Nursing, University of Michigan, Ann Arbor.

Barbara D. Evans MS, RN, GNP: Geriatric Nurse Practitioner, Rochester General Hospital, Rochester, NY; Ann E. Rogers PhD, RN: Assistant Professor, School of Nursing, University of Michigan.

Supported in part by Biomedical Research Support Grants from the School of Nursing and the School of Medicine at the University of Michigan and the Janet Gatherer Boyles Fund.

Address reprint requests to Ann E. Rogers, $P h D, R N$, School of Nursing, University of Michigan, $400 \mathrm{~N}$ Ingalls, Ann Arbor, MI 48109

Copyright (C) 1994 by W.B. Saunders Company

0897-1897/94/0702-0005\$5.0010 
Bunnell, 1990). Twenty-five percent of the healthy elderly subjects studied by Prinz in 1977 took a daytime nap, while $80 \%(n=8)$ of the healthy elderly subjects studied by Wauquier, van Sweden, Kerkhof, \& Kanphuisen (1991) took a daytime nap. Unfortunately, information is not available regarding the duration and timing of naps for these two early studies. A more recent study of very healthy elderly subjects (mean age 91.7 years) showed that subjects averaged about 30 minutes sleep during the daytime (Wauquier, van Sweden, Laqaay, Kemp, \& Kamphuisen, 1992). The number of daytime naps recorded during that study ranged from 0 to 5 , and the duration of daytime sleep ranged from 3 minutes to 76 minutes. Daytime naps accounted for $10 \%$ of the total sleep obtained by those very healthy elderly subjects. Nursing home residents usually sleep more during the daytime than persons living in their own homes (Bliwise et al., 1988).

Although numerous studies have shown there is a definite circadian (24-hour) rhythm for periods of decreased alertness and daytime napping (midafternoon) in adults, less is known about this rhythm in elderly persons. However, it is known that there are age-related changes in several circadian rhythms associated with sleep. For example, the excretion of melatonin, cortisol, and thyroidstimulating hormone (TSH) as well as production of leukocytes and neutrophils are altered in elderly persons (Bliwise, 1989b). In addition, core body temperatures in the elderly fall more rapidly on sleep onset and begin reversal at an earlier point in the sleep cycle than the core body temperatures of younger subjects (Zeplin, 1983). This phase advance in the temperature rhythm may contribute to the earlier onset of rapid eye movement (REM) sleep as well as to the early morning awakenings reported by many elderly persons (Bliwise, 1989b).

Although exposure to bright light is important in setting and maintaining sleep/wake rhythms, older persons, especially the institutionalized elderly, are exposed to far less sunlight than younger persons (Ancoli-Israel et al. 1991; Bliwise, 1989b). Alarm clocks, meals, or work schedules also are strong influences on circadian rhythms in human beings. When retired, individuals no longer have to adhere to regular schedules for arising and retiring, thus potentially reducing the number of environmental cues.
However, the individual's state of health may be more important than age-related alterations in the circadian timing system. For example, decreases in bladder capacity and changes in kidney function can lead to urinary frequency, nocturia, and sleep disruption (Matteson \& McConnell, 1988). In addition, joint pain and the discomfort associated with osteoarthritis and rheumatoid arthritis can make sleeping more difficult. Moreover, physical limitations on activity may encourage daytime napping (Bliwise, 1989b).

Dementias and mood also affect sleep. Studies have shown that demented subjects experience more sleep disruption than age-matched control subjects. There is a deterioration in the quality of nighttime sleep, with lowered sleep efficiencies, increased arousals, and more Stage 1 sleep as well as a redistribution of the sleep-wake cycle throughout the 24-hour day (Bliwise, 1989a; Morgan, 1987). Demented patients may or may not have less deep sleep than nondemented individuals (Bliwise, 1989a). However, demented individuals are 20 times more likely to nap during the day than nondemented subjects (Morgan, 1987).

Depression, which is the most common psychiatric disorder among older adults, causes distinct changes in sleep, particularly in aged individuals (Matteson \& McConnell, 1988). There is an increase in early morning awakenings and alterations in the timing of rapid eye movement (REM) sleep (Morgan, 1987). The sleep of older depressed patients is lighter, shorter, and more fragmented than that of younger depressed patients (Mendels \& Hawkins, 1968). Sleep efficiencies are lower in depressed elderly subjects compared with nondepressed elderly subjects (Mendels \& Hawkins, 1968).

Many medications can alter sleep. Methylxanthines, the drug family that includes caffeine and theophylline, are a common cause of insomnia. Caffeine is present in several nonprescription headache medications and dieting aids, as well as in coffee, tea, cocoa, chocolate, and soft drinks. The equivalent of just 2 to 4 cups of brewed coffee ( 200 to $400 \mathrm{mg}$ of caffeine) will increase the time it takes to fall asleep, increase the number of awakenings, and shift slow wave or deep sleep to the second half of the sleep period (Karacan et al., 1976). In addition, several drugs used to treat cardiovascular disease have been shown to disrupt nocturnal sleep. For example, the beta blockers 
such as propranolol are associated with insomnia, hallucinations, and nightmares (Roehrs \& Roth, 1989).

Although the number of persons over 65 years of age comprise only $13 \%$ of the United States population, they consume over $30 \%$ of all prescribed medication as well as an unknown percentage of over-the-counter medications (National Institutes of Health $[\mathrm{NIH}], 1991)$. In addition, the elderly consume more sleeping pills than any other age group; $35 \%$ to $40 \%$ of all the prescriptions written for sedatives and hypnotics were for persons over 65 years of age (Moran, Thompson, \& Mies, 1988). However, the safety and efficacy of these medications for treating sleep problems in the elderly has not been established (NIH, 1991). In fact, breathing difficulties due to obstructive sleep apnea can be exacerbated by the use of benzodiazepines such as flurazepam (Moran et al., 1988; Roehrs \& Roth, 1989). Additionally, studies have shown that the long-term use of sedativehypnotics or alcohol can induce a drug-related insomnia (Roehrs, Zorick, Sicklesteel, Wittig, \& Roth, 1983; Roth, Roehrs, \& Zorick, 1988). Finally, it is important to note that although elderly women have more subjective complaints about their sleep and receive more prescriptions for sedative and hypnotic medications than elderly men, polysomnographic recordings show that older women actually sleep better than older men (Bliwise, 1989b; Wauquier et al., 1992).

Although alcohol is frequently used to facilitate sleep, it is a relatively poor sedative-hypnotic (Moran et al., 1988). Not only docs alcohol disrupt nocturnal sleep, it is particularly dangerous for patients who have obstructive sleep apnea. Numerous studies show that alcohol worsens sleeprelated breathing problems in persons with obstructive sleep apnea, in asymptomatic individuals who have only occasional apneic pauses, and even among persons who snore but do not usually experience apneic pauses (Roehrs \& Roth, 1989).

Finally, alterations in sleep/wake patterns in the elderly may not simply be a function of biological aging or disease but may also reflect changing environmental demands and circumstances of the aging population (Woodruff, 1985). Retirement and changes in social patterns as well as the death of a spouse and/or close friends can lead to disturbances in sleep (NIH, 1991).

Numerous studies have documented that as one ages, sleep often becomes more fragmented, less consolidated, and less restorative than the sleep of earlier years. In addition to these developmental changes in sleep associated with aging, many other factors such as the presence of disease and the ingestion of drugs, particularly sedative-hypnotic medications and alcohol, often further disrupt the sleep of elderly persons. Although researchers have speculated that daytime napping may affect nocturnal sleep, little is known about the role that daytime naps may play in altering the sleep/wake patterns of older persons. Therefore, the purpose of this pilot study was to describe the 24-hour sleep/wake patterns, including daytime naps, of normal, healthy elderly individuals living independently at home. The following research questions were addressed: (a) What are the 24-hour sleep/ wake patterns of these older adults? and (b) What is the frequency, timing, and duration of any daytime naps?

\section{METHOD}

\section{Subjects}

To examine the sleep/wake patterns of healthy elderly subjects, a convenience sample of 14 subjects was recruited from the residents of a senior citizens' apartment complex located in a small midwestern city. All subjects had lived in that particular apartment complex for at least 6 months, and many had lived there for several years (5.1 \pm 5.2 years). The sample consisted of 13 women and 1 man ranging in age from 71 to 91 years $(81.2 \pm$ 5.9 years). All subjects were independent in their activities of daily living (ADLs) and were taking an average of 2.4 medications. Subjects were not allowed to consume alcohol during the 48-hour monitoring period. Excluded were those individuals who were taking hypnotics or tranquilizers, who had a history of sleep disorders and neurological or psychiatric problems, and who were not independent in their ADLs.

Letters were sent to all residents of a senior citizens' apartment complex describing the study and the eligibility criteria. Twenty residents returned a card requesting that they be contacted. The primary investigator contacted all potential subjects to verify that they met the criteria for participation. After reviewing the study procedure and obtaining written consent, the wrist actigraph was placed on 
the subject's nondominant wrist. Subjects also received written instructions at that time, and arrangements were made to have the actigraph and forms retrieved from the subject's apartment by one of the researchers.

\section{Instrumentation}

Forty-eight-hour recordings were made of each subject's sleep/wake pattern using a wrist actigraph (Ambulatory Monitoring Inc., Ardsley, NY). This small, lightweight, physical activity monitor senses, counts, and stores data about motor activity (Figure 1). Sleep and waking states are distinguished by the frequency and amplitude of movements. Studies comparing wrist actigraph recordings with standard polysomnographic recordings (PSG) have found reliability coefficients of .82 to .99 (Stampi \& Broughton, 1988; Zomer, et al., 1987). Software developed by Ambulatory Monitoring, Inc. was used to distinguish sleep from waking states and to calculate total sleep time, sleep latencies (time it took subject to fall asleep after going to bed), and other sleep statistics.

Subjective information was obtained from the activity diaries that all subjects kept while wearing the wrist actigraph. Subjects recorded their activities every 15 minutes (including the time of arising and retiring each day) and any medications that were taken during the recording period. Finally, subjects were asked to respond to several questions about the quality of their nighttime sleep, how they felt in the morning, and if they had napped, why they napped, and how they felt after napping.

Because seasonal variations in the intensity and duration of daylight can alter sleep/wake patterns, data collection for this study was confined to an 8-week period during the winter months. All recordings were obtained during the work week (Monday through Friday).

\section{RESULTS}

Because the sleep/wake patterns of these healthy elderly subjects were similar on days 1 and 2 of the 48-hour recording period, data from days 1 and 2 were combined. Although subjects spent slightly more than 7.5 hours in bed at night, they were asleep for just over 6 hours (Table 1). Subjects did not have difficulties falling asleep, but once asleep, had trouble remaining asleep. All subjects woke several times during the night (the fewest number of awakenings was 3 ) and usually stayed awake for 8 to 10 minutes each time they awakened (Figure 2). Sleep efficiencies (time asleep divided by the time spent in bed) ranged between $30.4 \%$ and $95.6 \%$.

The number of minutes spent asleep and awake for each hour was calculated. Over half of the subjects went to bed between 10:00 PM and 11:00 PM and got out of bed between 5:30 AM and 6:30 AM. Therefore, the hours between 11:00 PM and 5 AM, were examined in greater detail. More than $50 \%$ of the subjects were awake for at least some portion of each hour between 11 PM and 3 AM and then

Figure 1. The wrist actigraph used to collect data.

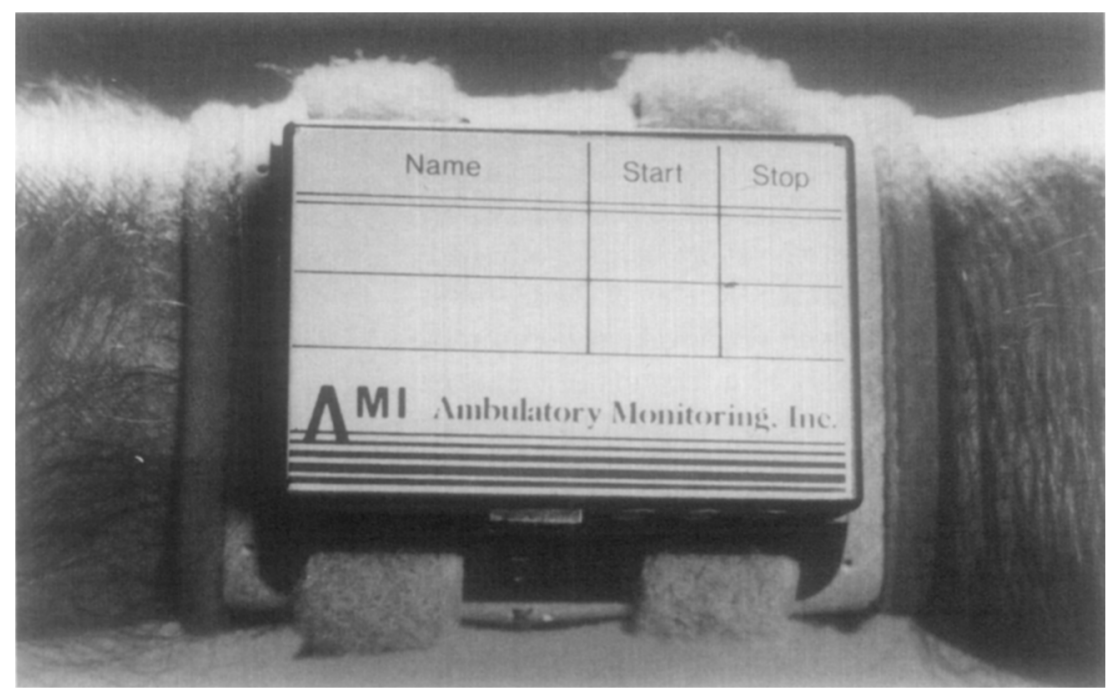


Table 1. Description of the Sleep/Wake Patterns of 14 Healthy Elderly Subjects Monitored for $\mathbf{4 8}$ Hours Using a Wrist Actigraph

\begin{tabular}{|c|c|c|c|c|}
\hline & \multicolumn{2}{|c|}{ Day 1} & \multicolumn{2}{|c|}{ Day 2} \\
\hline & Mean (Range) & so & Mean (Range) & SD \\
\hline \multirow[t]{2}{*}{ Total sleep time in 24-hr period (hrs) } & 7.0 & 1.7 & 7.5 & $1.8^{*}$ \\
\hline & $(3.98-10.6)$ & & $(4.13-11.7)$ & \\
\hline \multirow{2}{*}{ Total hours awake in 24-hr period } & 17.0 & 1.7 & 16.4 & 1.8 \\
\hline & $(13.4-20.0)$ & & $(12.0-19.9)$ & \\
\hline \multirow[t]{2}{*}{ Hours in bed at night } & 7.6 & .7 & 7.72 & 0.9 \\
\hline & $(6.5-8.8)$ & & $(6.1-9.1)$ & \\
\hline \multirow[t]{2}{*}{ Hours asleep at night } & 6.1 & 1.7 & 6.4 & 1.2 \\
\hline & $(2.1-8.2)$ & & $(3.8-7.6)$ & \\
\hline \multirow[t]{2}{*}{ Hours awake at night } & 1.5 & 1.3 & 1.4 & 1.1 \\
\hline & $(0.35-4.7)$ & & $(0.35-4.0)$ & \\
\hline \multirow[t]{2}{*}{ Sleep latency (min) } & 11.3 & 11.9 & 15.4 & 35.0 \\
\hline & $(0.7-47.7)$ & & $(1.7-136.7)$ & \\
\hline \multirow{2}{*}{ Hours awake after sleep onset } & 1.1 & 1.0 & 1.0 & 0.8 \\
\hline & $(0.2-3.2)$ & & $(0.2-3.0)$ & \\
\hline \multirow{2}{*}{ Number of awakenings } & 7.9 & 6.2 & 9.4 & 6.1 \\
\hline & $(3-24)$ & & $(3-23)$ & \\
\hline \multirow[t]{2}{*}{ Length of awakenings (mins) } & 9.5 & 7.8 & 6.5 & 3.0 \\
\hline & $(2.8-25.3)$ & & $(3.3-10.7)$ & \\
\hline \multirow[t]{2}{*}{ Sleep efficiency $(\%)$} & 79.7 & 19.5 & 82.4 & 13.3 \\
\hline & (30.4-95.3) & & $(54.1-95.6)$ & \\
\hline \multirow[t]{2}{*}{ Hours awake during daytime } & 15.6 & 0.9 & 15.1 & 1.1 \\
\hline & $(13.0-16.9)$ & & $(11.4-16.7)$ & \\
\hline \multirow[t]{2}{*}{ Minutes asleep during daytime } & 51.3 & 50.0 & 68.3 & 72.8 \\
\hline & $(0.0-145.0)$ & & $(0.0-247.0)$ & \\
\hline \multirow[t]{2}{*}{ Number of daytime naps } & 4.6 & 3.1 & 5.9 & 4.9 \\
\hline & $(0-10)$ & & $(0-17)$ & \\
\hline
\end{tabular}

${ }^{*} p<.05$.

awakened at least one more time between $4 \mathrm{AM}$ and 5 AM. Between the hours of $3 \mathrm{AM}$ and $4 \mathrm{AM}$, fewer subjects were awake.

Despite their fragmented and somewhat shortened nighttime sleep, subjects obtained most of their sleep during the nighttime hours. On average, of the total sleep recorded on Day $1,87.7 \%$ occurred at night, and on Day 2,84.8\% of the total sleep recorded occurred at night. However, exceptions were noted. Subject 10 , who obtained the least amount of sleep in a 24-hour period (3.98 hours on Day 1), obtained only $51 \%$ of her total

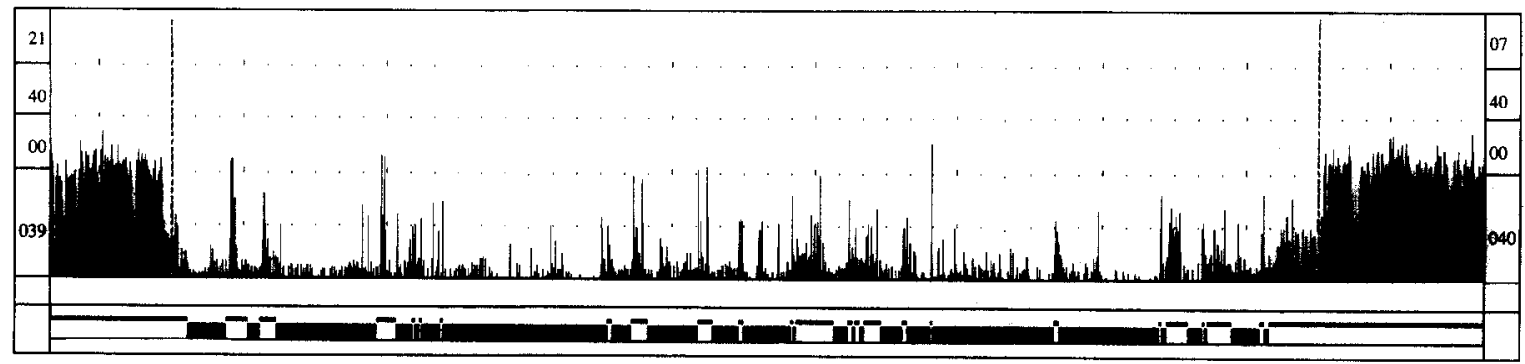

Figure 2. Actigraphic recording of a typical nights' sleep. The dashed vertical line on the left indicates when the subject went to bed, and the dashed vertical line on the right indicates when the subject got out of bed in the morning. —, wake, $\mathbf{E}$, sleep. 
sleep at night. Subject 7 , who slept more in 24 hours than any other subject (11.7 hours on Day 2 ), obtained $65 \%$ of her total sleep at night.

Although all subjects took at least one nap during the 48-hour recording period (12 of the 14 subjects took one or more naps on each day of the recording period), most subjects spent only a small percentage of the day asleep $(M=6.0 \%, S D=$ 4.1 , range $0 \%$ to $26.6 \%$ ). Naps were frequent but short. Subjects took, on average, $5.2 \pm 4.1$ naps per day, which lasted an average 9.6 minutes ( $S D$ $=8.2$, range 0 to 31.4 minutes). Total duration of daytime sleep averaged 59.8 minutes. Naps were longest in the afternoon. Daytime naps also were the most frequent during the afternoon. Of the 149 naps recorded, $42.9 \%$ occurred between 12 noon and 6 PM. Somewhat fewer naps occurred in the evening between 6 PM and 12 midnight (37.6\%), and slightly less than one fifth of the daytime sleep episodes (19.5\%) occurred during the morning (6 AM to 12 noon). Few subjects recorded these naps in their diaries. It was very common for subjects to indicate that they spent time watching television or reading before bedtime, when in fact, they were unable to remain awake for more than a few minutes at a time.

There were no significant correlations between age and duration of sleep at night $(r=.19)$, mean sleep efficiency $(r=.26)$, number of nocturnal arousals $(r=.13)$, or length of nocturnal arousals $(r=-.31)$ nor were there any significant relationships between age and duration of daytime sleep $(r=.21)$, number of daytime naps $(r=$ $.12)$, or mean duration of daytime naps $(r=.25$, Spearman's rho).

In order to more carefully examine the relationship between daytime napping and the nocturnal sleep of these healthy elderly subjects, three separate time periods were used. Night 1 , which was the time between subjects' retiring the first night and arising the next morning; daytime, which was the time between arising and retiring; and Night 2 , which was the time period between the subjects retiring the second night and arising the second morning. Although there was not a significant relationship between the number of nocturnal arousals on Night 1 and the number of daytime naps the following day $(r=.19)$, there were moderately strong but not statistically significant correlations between the number of nocturnal arousals and duration of sleep, both at night $(r=-.46)$ and during the daytime ( $r=-.34$, Spearman's rho).
As the number of nocturnal arousals increased, subjects tended to sleep less. There was only a very low association between the number of arousals on Night 2 and the number of naps taken during the daytime $(r=-.22)$. There was a moderately strong, but not statistically significant, correlation between the amount of sleep during the daytime and the duration of sleep on Night $2(r=-.47$, Spearman's rho). The duration of sleep on Night 2 tended to decrease as the duration of daytime sleep increased.

Despite frequent arousals, most subjects were satisfied with their nighttime sleep. Seventy-three percent rated their sleep as excellent or good. Only $27 \%$ of the sample rated their sleep as fair or poor. There was no significant relationship between subjects' satisfaction with their nocturnal sleep and most objective measures of sleep (minutes of nocturnal sleep, sleep latency, time awake after sleep onset, or the number of nocturnal arousals). As subject age increased $(r=.49)$ or the duration of nocturnal arousals increased $(r=.35$, Spearman's rho), subjects were more likely to rate their sleep as fair or poor. However, these correlations were not statistically significant.

Not all of the 9 subjects who reported napping responded to questions about their naps. The most frequent reason given for napping was fatigue ( $n$ $=5$ ). When awakening from a nap, most felt refreshed $(n=5)$, but two subjects reported no change in energy level, and one subject was still sleepy.

\section{DISCUSSION}

The major finding of this descriptive study was that nearly three fourths of the healthy elderly subjects studied were satisfied with their sleep despite frequent nocturnal arousals, reduced sleep efficiency, and a shortened nocturnal sleep. Secondly, daytime napping was more common than expected. Lastly, the extent of daytime napping was significantly underreported in the subjects' activity diaries.

Mean total sleep time, including daytime naps, was approximately 7 hours. Nocturnal sleep time averaged just over 6 hours, which is quite similar to the mean nocturnal sleep time reported for 10 Dutch subjects who underwent ambulatory polysomnographic monitoring for $\mathbf{4 8}$ hours (Wauquier, et al., 1991). All subjects took at least one daytime nap during the 48-hour monitoring period, which is much higher than previously reported (Prinz, 
1977; Wauquier et al., 1991, 1992). Daytime naps accounted for $12 \%$ to $15 \%$ of the total sleep obtained by these elderly subjects, which is somewhat higher than previously reported (Wauquier et al., 1992). Subjects took short (less than $10 \mathrm{~min}$ utes) but frequent naps. However, when these frequent short naps were added together, subjects slept, on average, an hour during the daytime. In contrast, the very healthy elderly subjects studied by Wauquier and his colleagues (1992) averaged slightly less than 30 minutes of sleep during the daytime.

It is worthwhile to note that subjects reported on average taking 1.79 naps per day in their activity diaries compared with an average of 5.2 naps per day recorded by the wrist actigraph. There are several possible explanations for this underreporting of daytime naps. Subjects may not have recorded short naps in their activity diaries because they were instructed to write down only those activities that lasted more than half of each 15-minute interval. A more likely explanation is that these subjects did not realize that they had dropped off to sleep for a few minutes while they were reading or watching television. Until a person has slept for at least 4 to 8 minutes or is awakened from Stage 2 sleep, their subjective awareness of having been asleep may be minimal (Bonnet \& Moore, 1982).

Other researchers have reported that the frequency of daytime napping increases with age and is most noticeable in advanced age (Hayter, 1983; Webb, 1989). However, there was no relationship between age and the frequency or duration of daytime napping in this sample of healthy elderly persons. It is possible that a relationship should have been detected between increasing age and daytime napping if the sample had been larger and had a wider age distribution. Although the age of subjects ranged from 71 to 91 years, 10 of the 14 subjects were between 75 and 87 years of age (mean age 81.2 \pm 5.9 years), and only 3 subjects $(21.4 \%)$ were less than 80 years old. However, when Metz and Bunnell (1990) surveyed 132 older adults, they found that neither gender nor age affected the prevalence of napping. Forty-eighthour ambulatory polysomnographic recordings have also confirmed that there are no differences in daytime napping behavior between elderly men and women (Wauquier et al., 1992).

It was not surprising that the largest percentage of naps recorded by the wrist actigraph occurred between noon and 6 PM (43\%). Numerous studies have shown that midafternoon is the favored time for napping among adults throughout the world (Broughton, Stampi, Dunham, \& Rivers, 1990; Webb \& Dinges, 1989). Even after retirement, when social demands for sustained wakefulness are substantially less, the predilection for afternoon sleep remains intact (Broughton, 1989; Wauquier et al., 1992). In fact, Wauquier and his colleagues (1992) reported that their very healthy elderly subjects who underwent 24 hours of ambulatory polsomnographic monitoring $(n=14)$ tended to nap at the beginning of the afternoon and during the early evening hours. Indeed, participants in this study were as likely to nap during the evening as during the afternoon (56 naps compared with 64 naps). Naps occurred during the early evening or just before retiring for the evening. Although a period of drowsiness before the nocturnal sleep period is rarely documented when sleep is monitored in a laboratory setting, Broughton and his colleagues (1990) have reported that most subjects monitored in the home environment will doze off while watching television or reading, then reawaken and go to bed for the night.

This group of relatively healthy, elderly subjects did not appear to be phase advanced (going to sleep and arising earlier than normal). However, without core body temperature measurements, it is impossible to accurately assess whether the timing of their nocturnal sleep periods was phase advanced or not. No subject retired before 9 PM, and only 2 subjects retired before 10 PM on one of the two nights of recording (10:10 and 10:45 PM). On four of the 28 nights recorded, subjects retired after midnight. However, most subjects retired between 10 and 11 PM. Subjects did awaken somewhat earlier than might be expected in a population not driven by work or school schedules. Three subjects arose between 5 and $6 \mathrm{AM}$ on both days of the study, and another arose at $5 \mathrm{AM}$ on the first day of the study. There were only 3 mornings when a subject slept later than 7:30 AM; the latest time for arising was 8:20 AM.

Similar to other studies, a great deal of variability between subjects in terms of times for arising and retiring, number and duration of nocturnal arousals, number and duration of daytime naps, and TST was identified (Hayter, 1983; Wauquier et al., 1991). However, sleep patterns were fairly consistent for each subject. Those subjects who tended to go to bed later on Day 1 usually retired later on Day 2. Likewise, subjects who had fre- 
quent nocturnal arousals or took frequent daytime naps also did so on Day 2.

No first-night effect was documented. There were no significant differences in time in bed, duration of nocturnal sleep, sleep latency, duration of waking after sleep onset, the number and duration of nocturnal arousals, and sleep efficiency between the first and second nights of the recording period. Although the first-night effect is less likely to occur when ambulatory monitoring equipment is used in the home environment (Broughton et al., 1990; Wauquier et al., 1992), Wauquier and his colleagues (1991) noted that their elderly subjects fell asleep quicker, had higher sleep efficiencies, and slept longer during the second night of ambulatory monitoring. They used ambulatory electroencephalogram (EEG) monitors to collect their data, not wrist actigraphs. It is possible that the larger and heavier ambulatory EEG monitors were more disruptive to sleep patterns than the wrist actigraphs used for this study.

Future studies should include larger and more representative samples of elderly persons drawn from an entire community. A broader range of ages would be more desirable as well as the inclusion of more elderly men in the study. Sleep patterns from elderly subjects with varying degrees of health also should be examined. Because selection criteria excluded those with diagnosed sleep disorders, neurological and psychiatric problems, as well as those subjects who were taking any psychotropic medications (including sedativehypnotics), subjects in this study were likely to be healthier and have fewer sleep complaints compared with most other elderly persons.

Although knowledge about changes in sleep/ wake patterns associated with aging is still somewhat limited, nurses need to be aware that the sleep/wake patterns of healthy elderly persons may be different from those of younger persons. Data from this and other studies suggest that frequent nocturnal arousals, a somewhat shortened nocturnal sleep period, and reduced sleep efficiencies are normal changes associated with aging. "Whether an aged individual views his or her $75 \%$ sleep efficiency as insomnia or merely accepts this as a normal part of aging may depend largely on that individual's perspective on growing old and what that means to him or her"' (p. 40, Bliwise, 1993). Indeed, the majority of the sample were satisfied with the quality of their nocturnal sleep. If an elderly person is dissatisfied with their nocturnal sleep and physiological causes such as pain, sleep apnea, or periodic leg movements have been ruled out, behavioral techniques such as sleep restriction therapy can be quite effective in consolidating and improving nocturnal sleep (Engle-Friedman, Bootzin, Hazlewood, \& Tsao, 1992). The prescription of sedative-hypnotic medications to reverse these normal developmental changes is rarely appropriate, often unnecessary, and potentially dangerous.

Although the avoidance of daytime napping is often recommended to improve nighttime sleep, there is no reason to discourage daytime napping (Dinges, 1989; Hayter, 1983; Metz \& Bunnell, 1990). There was a slight tendency for increases in the amount of sleep during the daytime to be related to decreases in the duration of nocturnal sleep. However, this relationship was not significant. Nor were there any significant correlations between daytime napping and noctumal sleep duration, sleep fragmentation, and wake after sleep onset in the Wauquier et al. (1992) study of 14 very healthy elderly persons. All of subjects in this study napped, and the majority of the healthy elderly subjects studied by Wauquier and his colleagues $(1991,1992)$ napped during the day.

When assessing the sleep/wake patterns of elderly clients, nurses should remember that their elderly clients are very likely to underreport the number of daytime naps. Information obtained when interviewing a client may be biased by the client's own preconceptions or by their perception of the health care provider's interests or bias (Douglas, Carskadon, \& Houser, 1990). Data obtained from questionnaires and sleep diaries also is likely to be incomplete or inaccurate (Buysse et al., 1991; Douglas et al., 1990). The client, particularly one who takes frequent, short naps, may simply not remember napping during the day (Douglas et al., 1990). If more accurate information is needed regarding daytime napping, objective rather than subjective measures must be used.

Finally, it is worth remembering and of clinical importance that despite their somewhat shortened and fragmented nocturnal sleep, the majority of our elderly subjects were pleased with their nighttime sleep and reported being refreshed by their daytime naps. In fact, Buysse and his colleagues (1991) believe that elderly persons in good psychological and physical health may alter their perception of sleep quality to accommodate the sleep disturbances that accompany aging. 


\section{REFERENCES}

Ancoli-Israel, S., Kripke, D.F., Jones, D.W., Parker, L., \& Hanger, M.A. (1991). 24-Hour sleep and light rhythms in nursing home patients. Sleep Research, 20A, 410.

Bliwise, D.D. (1989a). Dementia. In M.H. Kryger, T. Roth, \& W.C. Dement (Eds.), Principles and practice of sleep medicine (pp. 358-363). Philadelphia, PA: Saunders.

Bliwise, D.L. (1989b). Normal aging. In M.H. Kryger, T. Roth \& W.C. Dement (Eds.), Principals and practice of sleep medicine (pp. 24-29). Philadelphia, PA: Saunders.

Bliwise, D.L. (1993). Sleep in normal aging and dementia. Sleep, 16, 40-81.

Bliwise, D., Pursley, A., \& Dement, W.C. (1988). Day/ night relationships of observed sleep/wakefulness in aged nursing home residents. Sleep Research, 17, 72.

Bonnet, M.H. \& Moore, S.E. (1982). The threshold of sleep: Perception of sleep as a function of time asleep and auditory threshold. Sleep, 5, 267-276.

Broughton, R.J. (1989). Chronological aspects and models of sleep and napping. In D.F. Dinges \& R.J. Broughton (Eds.), Sleep and alertness: Chronobiological, behavioral and medical aspects of napping (pp. 71-98). New York, NY: Raven Press.

Broughton, R.J., Stampi, C., Dunham, W., \& Rivers, M. (1990). Ambulant monitoring of sleep-wake state, core body temperature, and body movement. In L.E. Milcs \& R.J. Broughton (Eds.), Medical monitoring in the home and work environment (pp. 139-150). New York, NY: Raven Press.

Buysse, D.J., Reynolds, C.F. Monk, T.H., Hoch, C.C., Yeager, A.L., \& Kupfer, D.J. (1991). Quantification of subjective sleep quality in healthy elderly men and women using the Pittsburgh Sleep Quality Scale (PSQI). Sleep, 14, 331-338.

Dinges, D. (1989). Napping patterns and effects in human adults. In D. Dinges \& R. Broughton (eds.), Sleep and alertness: Chronobiological, behavioral and medical aspects of napping (pp. 171-204). New York, NY, Raven Press.

Douglas, A.B., Carskadon, M.A., \& Houser, R. (1990). Historical data base, questionnaires, sleep and life cycle diaries. In L.E. Miles \& R.J. Broughton (Eds.), Medical monitoring in the home and work environment (pp 17-28). New York, NY: Raven Press.

Engle-Friedman, M., Bootzin, R.R., Hazlewood, L., \& Tsao, C. (1992). An evaluation of behavioral treatments for insomnia in the older adult. Journal of Clinical Psychology, 48, $77-90$

Hayter, J. (1983). Sleep behaviors of older persons. Nursing Research, 32, 242-246.

Karacan, I., Thornby, J.I., Anch, A.M., Booth, G.H., Williams, R.L., \& Salis, P.S. (1976). Dose-related sleep disturbances induced by coffee and caffeine. Clinical Pharmacology and Therapeutics, 20, 682-689.

Matteson, M., \& McConnell, E. (1988). Gerontological nursing: Concepts and practice. Philadelphia, PA: Saunders.

Mendels, J., \& Hawkins, D.R. (1968). Sleep and depression: Further considerations. Archives of General Psychiatry, 19, 445-452.

Metz, M.E., \& Bunnell, D.E. (1990). Napping and sleep disturbances in the elderly. Family Practice Research Journal, 10, 47-56.

Moran, M.G., Thompson, T.L., \& Mies, A.S. (1988). Sleep disorders in the elderly. American Journal of Psychiatry, 145, 1369-1377.

Morgan, K. (1987). Sleep and aging. Baltimore, MD: The Johns Hopkins University Press.

National Institutes of Health concensus development conference statement (1991): The treatment of sleep disorders in older people. Sleep, 14, 169-177.

Pressman, M., \& Fry, J. (1988). What is normal sleep in the elderly? Clinics in Geriatric Medicine, 4, 71-81.

Prinz, P. (1977). Sleep patterns in the healthy elderly: Relationship with intellectual function. Journal of Gerontology, 32, 179-186.

Roehrs, T.A., \& Roth, T. (1989). Sleep disorders in the elderly. Clinics in Geriatric Medicine, 5, 395-494.

Roehrs, T.A., Zorick, F., Sicklesteel, J., Wittig, R., \& Roth, T. (1983). Age-related sleep-wake disorders at a sleep disorder center. Journal of the American Geriatric Society, 31 , 364-370.

Roth, T., Roehrs, T.A., \& Zorick, F. (1988). Pharmacological treatment of sleep disorders. In R.L. Williams, I. Karacan, \& C.A. Moore (Eds.), Sleep disorders: Diagnosis and treatment. New York: Wiley.

Stampi, C. \& Broughton, R. (1988). Ultrashort sleep-wake schedule: Detection of sleep state through wrist actigraph measures. Sleep Research, 17, 100.

Wauqueir, A., van Sweden, B., Kerkhof, G.A., \& Kamphuisen, H.A.C. (1991). Ambulatory first night sleep effect recording in the elderly. Behavioral Brain Research, 42, 7-11.

Wauqueir, A., van Sweden, B., Lagaay, A.M., Kamp, B., \& Kamphuisen, H.A.C. (1992). Ambulatory monitoring of sleep-wakefulness patterns in healthy elderly males and females (>88 years): The "senieur" protocol. Journal of the American Geriatric Society. 40, 109-114.

Webb, W. (1989). Development of human napping. In D. Dinges \& R. Broughton (Eds.), Sleep and alertness: Chronobiological, behavioral, and medical aspects of napping (pp.3152). New York, NY: Raven Press.

Webb, W.B. \& Dinges, D.F. (1989). Cultural perspectives on napping and the siesta. In D. Dinges \& R. Broughton (Eds.), Sleep and alertness: Chronobiological, behavioral, and medical aspects of napping (pp. 247-265). New York, NY: Raven Press.

Woodruff, D. (1985). Arousal sleep and aging. In J.E. Birren \& K.W. Schaie (Eds.), Handbook of the psychology of aging (pp. 261-295). New York NY: Van Nostrand Reinhold Company.

Zeplin, H. (1983). Normal age related changes in sleep. In M. Chase \& E. Weitzman (Eds.), Sleep disorders: Basic and clinical research (pp. 431-444). New York, NY: Spectrum Publications.

Zomer, J., Pollack, I., Tzischinshy, O., Epstein, R., Alster, J., \& Lavie, P. (1987). Computerized assessment of sleep. Sleep Research, 16, 592. 